\title{
Acute Carotid Dissection Causing Acute Ischemic Stroke Due to Cocaine Sniffing
}

\author{
Nilesh Chaudhary ${ }^{1}$ \\ 1Department of Neurology, Dr. L.H. Hiranandani Hospital, Powai, \\ Mumbai, Maharashtra, India
}

Int J Recent Surg Med Sci 2021;7:34-36.

\begin{abstract}
Address for correspondence Nilesh Chaudhary, MBBS, DNB, Department of Neurology, Dr. L.H. Hiranandani Hospital, Powai, Mumbai, Maharashtra, India (e-mail: nilmint@gmail.com).
\end{abstract}

\begin{abstract}
Introduction Mechanism of acute ischemic stroke in young adults due to acute carotid dissection can be due to cocaine sniffing, as it is sympathomimetic for cranial circulation.

Methods A 24-year-old female presented with acute onset of right-sided neck pain and transient left hemiparesis lasting for 15 minutes after sniffing of cocaine. She had another episode of left hemiparesis with dysarthria (with National Institutes of Health Stroke Scale [NIHSS] score 7), which did not recover after second sniffing of cocaine. Her urgent magnetic resonance imaging of the brain and computed tomography angiography showed acute ischemic stroke in the right middle cerebral artery territory with acute right proximal carotid dissection with thrombus. She was having high blood pressure during presentation, which was optimized with labetalol, and was started on low-molecular-weight heparin and antiplatelet and statins. She improved gradually and her NIHSS score at discharge was 4.
\end{abstract}

Keywords

- stroke

- carotid dissection

- cocaine sniffing

- young stroke

Discussion Cocaine, due to its sympathomimetic properties, causes sudden vasoconstriction; acute surge in blood pressure shortly after intake of cocaine may increase shear stress in the aortic wall, leading to a disruption of the intimal layer and a dissection with an intramural hematoma that subsequently may rupture into the lumen and cause acute ischemic stroke. In our case clinical history was unique in that episodes of cocaine sniffing caused progressive carotid dissection and subsequent stroke. Treatment needs optimal control of blood pressure and anticoagulation and antiplatelet medication.

Conclusion Cocaine sniffing is one of the rare causes of acute ischemic stroke in young adults, and the mechanism can be acute carotid dissection.

\section{Introduction}

Cocaine is an illegal recreational drug that has strong sympathomimetic properties and that is responsible for acute malignant hypertension and subsequent toxic neurovascular and cardiovascular complications.
We are reporting this case to establish a relationship between cocaine use and risk of stroke, with reference to stroke onset and its relationship with timing and route of cocaine use.
DOI https://doi.org/

$10.1055 / \mathrm{s}-0040-1721548$

ISSN 2455-7420. (c) 2020. Medical and Surgical Update Society.

This is an open access article published by Thieme under the terms of the Creative Commons Attribution-NonDerivative-NonCommercial-License, permitting copying and reproduction so long as the original work is given appropriate credit. Contents may not be used for commercial purposes, or adapted, remixed, transformed or built upon. (https://creativecommons.org/licenses/by-nc-nd/4.0/).

Thieme Medical and Scientific Publishers Pvt. Ltd. A-12, 2nd Floor, Sector 2, Noida-201301 UP, India 


\section{Case Report}

A 24-year-old female presented to the emergency room with significant neck pain for the last 1 week with left hemiparesis with dysarthria for the last 1 day.

On further history taking, she revealed that she had transient left hemiparesis 1 week back, which lasted few hours, but she had not approached medical facility.

She was not diabetic or hypertensive, had no family history, and no history of trauma.

On examination she was conscious and cooperative, with regular pulse rate of $90 / \mathrm{min}$ and blood pressure of $180 / 90 \mathrm{mmHg}$, which was managed with labetalol as her blood pressure remained around 130/75 $\mathrm{mmHg}$.

She was having dysarthria, left upper motor neuron facial palsy, and left hemiparesis with National Institutes of Health Stroke Scale score of 7. Magnetic resonance imaging of her brain revealed acute infract with hemorrhagic transformation in right lentiform nucleus and corona radiata. She also had multiple small acute infracts in right frontal, parietal, and superior temporal regions with occlusion of posterior division of M3 segment of right middle cerebral artery and right proximal internal carotid artery (ICA) dissection.

Computed tomography angiography of the brain was performed, which confirmed proximal right ICA dissection with adherent long-segment thrombus in proximal lumen causing 50\% narrowing. Carotid Doppler showed distal portion of floating thrombus. She started on low-molecular-weight heparin and high-dose statins with antiplatelets.

Her serum $\mathrm{Hb}$ was 8.3, serum homocysteine was normal, lipid profile was normal, and 2D echocardiography was also normal.

Work up for anemia was showing pattern of iron deficiency anemia.

As the patient was a young person, we were not able to identify the cause and hence we decided to go back in history again.

So, after direct questioning we got the information that she is a cocaine abuser for the past 2 years and takes cocaine once in 2 weeks.

She used to take cocaine by inhalation (sniffing); recently she had changed the quality of cocaine (as per the history given by the patient), which was more costly and gave her more desirable effects, as per the patient. As shown in - Fig. 1,

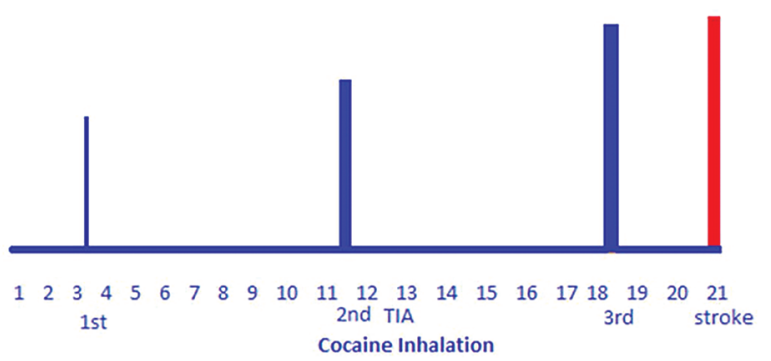

Fig. 1 Correlation of cocaine sniffing and onset of neurodeficits. Blue line indicates TIA and red line indicates acute ischemic stroke. Acute stroke thickness of line indicates that higher the thickness, higher the sniffing dose. TIA, transient ischemic attack. on day 3 she had her regular sniffing; later she sniffed on the night of day 11 and got headache with severe neck pain and had transient left hemiparesis (transient ischemic attack) on day 12 . On day 18 , at early morning 4 AM, she had another puff of cocaine; this time she consumed little large quantity and noticed neck pain again during this puff. She was asymptomatic on day 19, but on day 20 she developed left hemiparesis with dysarthria.

There was temporal correlation between cocaine sniffing and onset of neck pain and sequential neurodeficits, suggesting inhalation of cocaine contributing to carotid dissection.

\section{Discussion}

This was a case of acute ischemic stroke dysarthria due to right proximal carotid artery dissection. We have observed temporal correlation between cocaine sniffing and dissection.

Cocaine is known to affect the vascular system by its sympathomimetic properties, which is responsible for various neurovascular and neurological complications like headache, ischemic stroke, cerebral or subarachnoid hemorrhage, and seizures. ${ }^{1}$

Pharmacological action of cocaine is by blocking the reuptake of noradrenaline and dopamine from the synaptic cleft into presynaptic neurons. ${ }^{2}$

This is responsible for increased levels of noradrenaline in the synaptic cleft, which in turn activates postsynaptic $\alpha$-adrenergic and $\beta$-adrenergic receptors. ${ }^{3}$

There may be acute onset release of noradrenaline into the synaptic cleft by cocaine. Activation of $\alpha-1$ receptors in blood vessels causes elevation of the systemic blood pressure through vasoconstriction. Cocaine also causes increased ventricular contractility contributing to increase in blood pressure. ${ }^{1,3}$

So, treatment of blood pressure needs use of combined $\alpha$ and $\beta$ blockers.

The sudden shooting in blood pressure shortly after intake of cocaine may increase shear stress on the aortic wall, subsequently causing a disruption of the intimal layer and a dissection. ${ }^{4}$

More infrequently, the stress may produce an intramural hematoma that subsequently may rupture into the lumen. ${ }^{5}$

Cocaine-induced aortic dissection secondary to inhalation and insufflation is well documented in various short case series. ${ }^{3,5}$

Cocaine-induced strokes are ischemic due to vasospasm of large cerebral arteries followed by cerebral vasculitis, and cardioembolic due to cocaine-induced cardiomyopathy. ${ }^{6}$

There are case reports of carotid dissection due to cocaine sniffing; ${ }^{6-8}$ our case was similar to those. Unique feature was history of sniffing followed by immediate neck pain, suggesting impending carotid dissection.

\section{Conclusion}

Proximal carotid dissection is one of the rare causes of acute ischemic stroke in young adults due to cocaine sniffing, as cocaine is a vasoconstrictor. 
Malignant hypertension in cocaine sniffing needs treatment with combined alpha and beta blockers.

\section{Conflict of Interest}

None declared.

\section{References}

1 Tsatsakis A, Docea AO, Calina D, et al. A mechanistic and pathophysiological approach for stroke associated with drugs of abuse. J Clin Med (Lond) 2019;8(9):12-95

2 Levine SR, Brust JC, Futrell N, et al. Cerebrovascular complications of the use of the "crack" form of alkaloidal cocaine. N Engl J Med 1990;323(11):699-704
3 Schmidt HD, Pierce RC. Cocaine-induced neuroadaptations in glutamate transmission: potential therapeutic targets for craving and addiction. Ann NY Acad Sci 2010;1187:35-75

4 Farooq MU, Bhatt A, Patel M. Neurotoxic and cardiotoxic effects of cocaine and ethanol. J Med Toxicol 2009;5(3):134-138

5 Neri E, Toscano T, Massetti M, Capannini G, Frati G, Sassi C. Cocaine-induced intramural hematoma of the ascending aorta. Tex Heart Inst J 2001;28(3):218-219

6 Treadwell SD, Robinson TG. Cocaine use and stroke. Postgrad Med J 2007;83(980):389-394

7 Behrouz BR. Disección de la arteria carótida interna después del consumo de cocaína S. Rev Neurol 2015;61:46

8 Meghani M, Siddique MN, Bhat T, Samarneh M, Elsayegh S. Internal carotid artery redundancy and dissection in a young cocaine abuser. Vascular 2013;21(4):243-245 